\title{
Panoramic Image Stitching with Efficient Brightness Fusion Using RANSAC Algorithm
}

\author{
Jungpil Shin ${ }^{1}$, Md Abdur Rahim², Keun Soo Yun ${ }^{* 3}$ \\ ${ }^{1,2}$ School of Computer Science and Engineering, the University of Aizu, Aizu-wakamatsu, Fukushima, Japan, 965-8580 \\ *3, Corresponding author Department of Computer and Information, Ulsan College, Ulsan, Korea \\ *Corresponding authorE-mail: ksyun@uc.ac.kr
}

\begin{abstract}
Background/Objectives: Image stitching can enhance the picture very pleasant by modifying and mixing the different aspects.Therefore, we present panoramic image stitching with efficient brightness fusion which is challenging in different bright sequences taken from different angles.

Methods/Statistical analysis:For the problem of brightness, the input image is mixed with sequential images in different brightness.In this works, we proposed atechnique that blends multiple brightness using simple quality measures like color, saturation and contrast.The resulting image quality is good, and most important thing is, the method is efficient since it is simple. Thanthe resulting fused images is applied for panorama image stitching. We used multiband blending to prevent the blurring, and BRIEF (binary robust independent elementary features) method for feature descriptors. We solved the multi-image matching problem using Hamming Distanceusing the binary string based descriptors which is most similar features compare with the second most similar images. We proposed FLANN based matcher to get the more accurate results for using large datasets. We estimate Homography with the matching images using RANSAC algorithms.

Findings: An effective structure is performed when we are able to resolve the brightness correction in expose too much or expose for too short a time and the appearance ghost. To solve the unification of brightness, we have collected the input images in different exposures, and selection of the good parts of each picture to an input image for stitching. We removed the blurring from input images, and solved multi-image matching using Hamming distance method. We found better results comparing other methods. For large dataset, we used FLANN based matcher, and estimated the Homography using RANSAC algorithm.

Improvements/Applications:We have shown the performance of panoramic image stitching with efficient brightness fusion. We performed stitching with high regulationimages. Finally, we were able to create a panoramic image with efficient brightness fusion.
\end{abstract}

Keywords: Panorama, Brightness Fusion, Image stitching, RANSAC Algorithm, Homography

\section{Introduction}

Image stitching is the combination of resembling photographic images with the view of coordinated areas for creating a segmented panorama or high-quality image.Comprehensively, computer software is used to perform for image stitching. Therefore, it requires the accurate overlap in the identical exposure to be made to produce the seamless results, although some stitching algorithms [1] are actually used to imitate HDR (High Dynamic Range) imaging in different types of images [2]. However, the main problem to tackle, the presence of distortion of lens, visible and the differences of being exposure.For panoramic stitching, the ideal set of images will be able to exceed the distortion of the lens and have an overlap appropriate amount for adequate identifiable features [3].

In this study, we present a strategy that reflects the quality of color, saturation, and contrast, adding brightness correction modes, and we produce high-quality results for rendering simultaneous output panoramas using multi-band.The concept of image stitching is primarily used to prospect the panorama scenes from multiple images of single images. Image stitching method can be grouped into two general ways: [4-5] i. direct techniques and ii. feature based techniques. Direct comparison measure with the intensity of all the pixels in the pictures with each other, while feature-based strategies for determining a relationship between images through processed images.In our system, we used the hamming distance mechanism to match the input image feature. The overall procedure of the proposed system is shown in Figure 1.

The paper is organized as follows: Section 2 describes previous research studies. In section 3 we propose the brightness fusion and key point detection methodology. Section 4 explains the feature matching procedure by Hamming distance of the input image. In section 5,we propose the image matching and verification methodology. In section 6 describesthe experimental results and discussions. In section 7 , we present conclusions and ideas for future work and section 8 , mention the study related references.

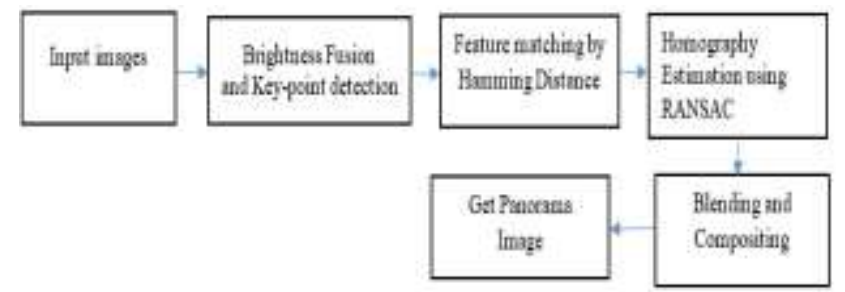

Figure 1: Overall process of the system. 


\section{Related Works}

In this section, we have explained many of these researchers in recent years, therefore, introducing relevant work, we cited some research as the following example:

In [6], M. Brown and D. G. Lowedescribed a systematic method based on an unchangeable feature for storing fully automated panoramic images. It was sensitive to input image sequence, orientation, scale, and illumination. They have a problem with a multi-image matching and using final matches using all the unique local features in all the images.Z. Wanget al. found a looping path problem in error accumulation and proposed a multi-image stitching method based on a graph model [7]. In [8], S. Pravenaa and R. Menaka discussed the calibration, registration and blending method to perform an image stitching operation. S. Mistry and A. Patel explained the Harris corner detection algorithm in details, and also RANSAC algorithm to remove the outliers from the two images [9]. D. G. Lowe presented a method to find indistinguishable characteristics from images that can be used to reliably assimilate different views of an object or scene. [10]. Color image mosaics formed [11] in image registration and image fusion that was presented an advanced RANSAC algorithm. A histogram matching based image stitching described in [12]. A new fusion system was proposed on a local domain [13] using the propagated image filter. To calculate the weight map of each input image by image filter and gradient domain post-processing process.According to the stitching line to seamlessly stitch the images based on an energy map described in [14]. This method integrated many of superior features at the same time. Y. Tang et al.proposed a spontaneous strategy based on a map that is essentially a combination of a gradient map which represents the presence of structures and the attraction of a region and the presence of maps [15].

In the above research, researcher achieved optimal performance. How, in the most researches, explained the image stitching based on the energy map, ordering, adaptation, scale, and illumination etc.Therefore, in our research shows how to perform the efficient brightness fusion between input images, and to improve the accuracy of the system by using binary string based descriptors, and FLANN based matchers to get more accurate results and finally, get a panorama image using RANSAC algorithm.

\section{Brightness Fusion and Key-Point Detection}

\subsection{Brightness Image Fusion}

Image Fusion is a combination of pictures of one or more images into a single image of a clearly informative picture with interest information.We suggested a solution is a brightness based on the quality measurements such as color, saturation, and contrast [16].It is used for the combination of multiple resolution in order to smooth the variations of brightness in the perfect image.We have collected a lot of images taken with different exposures for the same scene and the image is fully connected. In order to achieve this, we must ensure that our cameras position should be adjusted or there will be no biased camera movement.In this study, we used the registration algorithm [17] for the resemble of potentially combined regions of these images.For brightness fusion, the poor portion should be excluded and the good portion should be kept according to the order of multi-brightness images. We measured the brightness on three strategies, and create a weight map. Therefore, we perform weight-based composing of multiple images. The image fusion process is depicted in Figure 2

\subsubsection{Method of Brightness Measurement}

We performed the following steps to determine the weight, whether it is well-expressed for measuring the stack images.
However, the areas of the images are under or overexposed, we obtain less weight when the brightly colored area should be composed.

Measurement of Exposedness(E): In this method, we have given importance to each intensity, on which it uses Gauss's curve to close to $0.4 \exp \left(-\frac{(\mathrm{i}-0.4)^{2}}{2 \sigma^{2}}\right)$. Weused Gausscurve separately in this system.

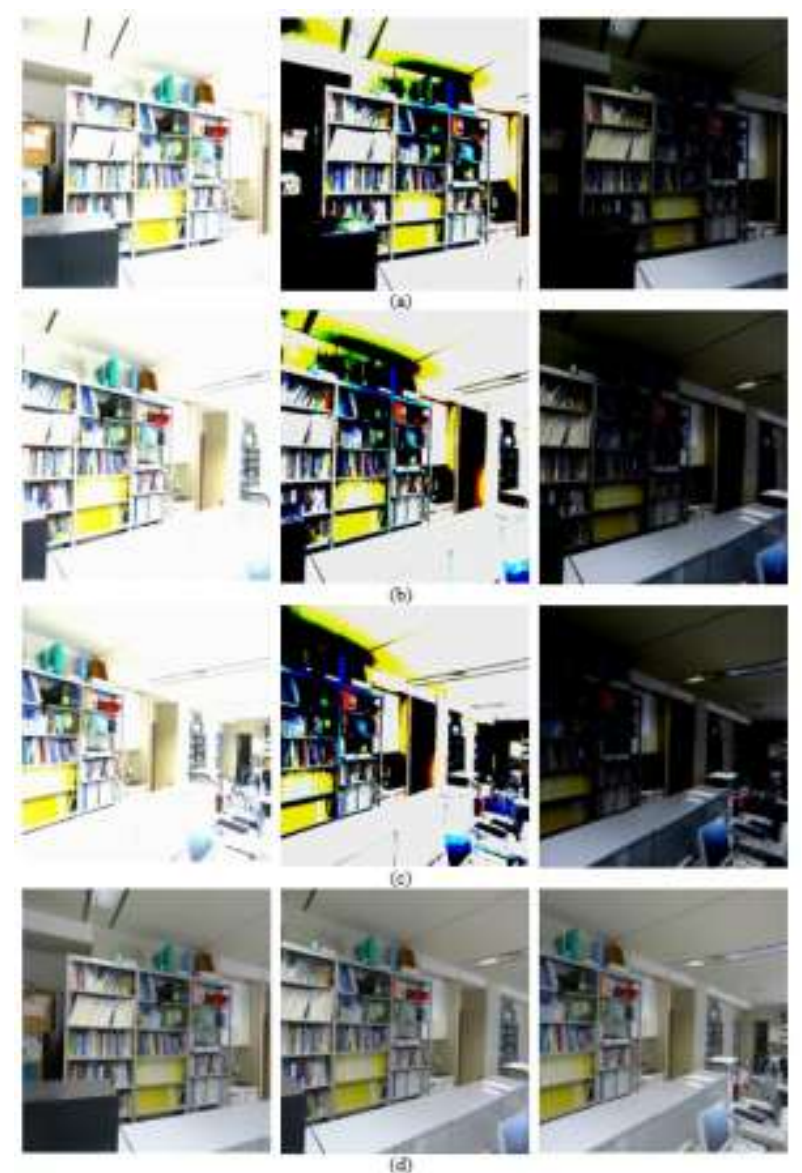

Figure2: The sequence of input images in different brightness are (a), (b), (c), and the result of fused images are in (d).

Measurement of Contrast (C): We used the Laplacian filter of each image and received an absolute quality filter response. Due to the Laplacian filter, it will provide a high weight for the main part of the edges.

Measurement of Saturation (S): According to the photographic measurement, when an image is exposed for a long time, the color will be desaturated. We consider a saturation measurement by standard deviation computing between $\mathrm{R}, \mathrm{G}$ and $\mathrm{B}$ channels in each pixel.

We obtained the measurement using a linear power function:

$W_{i j, k}=\left(C_{i j, k}\right)^{w_{C}}\left(S_{i j, k}\right)^{w_{S}}\left(E_{i j, k}\right)^{w_{E}}$

$w_{\mathrm{C}}, w_{\mathrm{S}}, w_{\mathrm{E}}$ is is the symbol of measurement $\mathrm{C}, \mathrm{S}, \mathrm{E}$, and $\mathrm{i}, \mathrm{j}$, kreference to the pixel ofi, $\mathrm{j}$ in the k-th image. Therefore, the measurement can be calculated when the exponent value of $w$ is zero.

\subsubsection{Image Fusion using Laplacian}

In ordering serialized images $\hat{I}_{i j, k}$, we can get the perfect image Iwith an overlay composition of input images:

$I_{i, j}=\sum_{k=1}^{N} W_{i j, k} \hat{I}_{i j, k}$ 
the sequence of k-th input image is $\hat{I}_{k}$. However, the equation 2 seems to be a problem when it is applied. Due to their exposure times, the images are combined with assorted intensity. As a solution to this problems, we performed an image mixing seamlessly with an alpha mask, and used Laplacian method for image decomposition. However, this method is useful for avoid seams built in multi-resolution.

\subsection{Key-Point Detection}

The detection of features needs to be automatically identified in the image. The image requires a strong addition to estimating the transition needed for image alignment. We proposed BRIEF method to find binary string easily without finding feature descriptions. Therefore, itaccepts a smooth image patch, and selects a set of ${ }^{n} d_{(x, y)}$ location is a unique way to pair. However, the comparisons of some pixel intensities measured to these position. Since, thepair of the first location are $\boldsymbol{P}$ and $\boldsymbol{q}$. We found the result is 1 when $I(\boldsymbol{p})<I(q)$, otherwise the results calculated 0 . Finally, we applied this method for all the ${ }^{n} \boldsymbol{d}$ pairs location and we obtained the ${ }^{n} \boldsymbol{d}$-dimensional bit string. We can use 128,256 or 512 for ${ }^{n} \boldsymbol{d}$-dimensional bit string, but 256 can be used as a default value.Therefore, we used BRIEF method which is a faster for matching and calculation of feature descriptor. Since, it provides high acceptance rates when it does not have a large inplane rotation. The key-point detection exhibited using BRIEF method is shown in Figure 3.
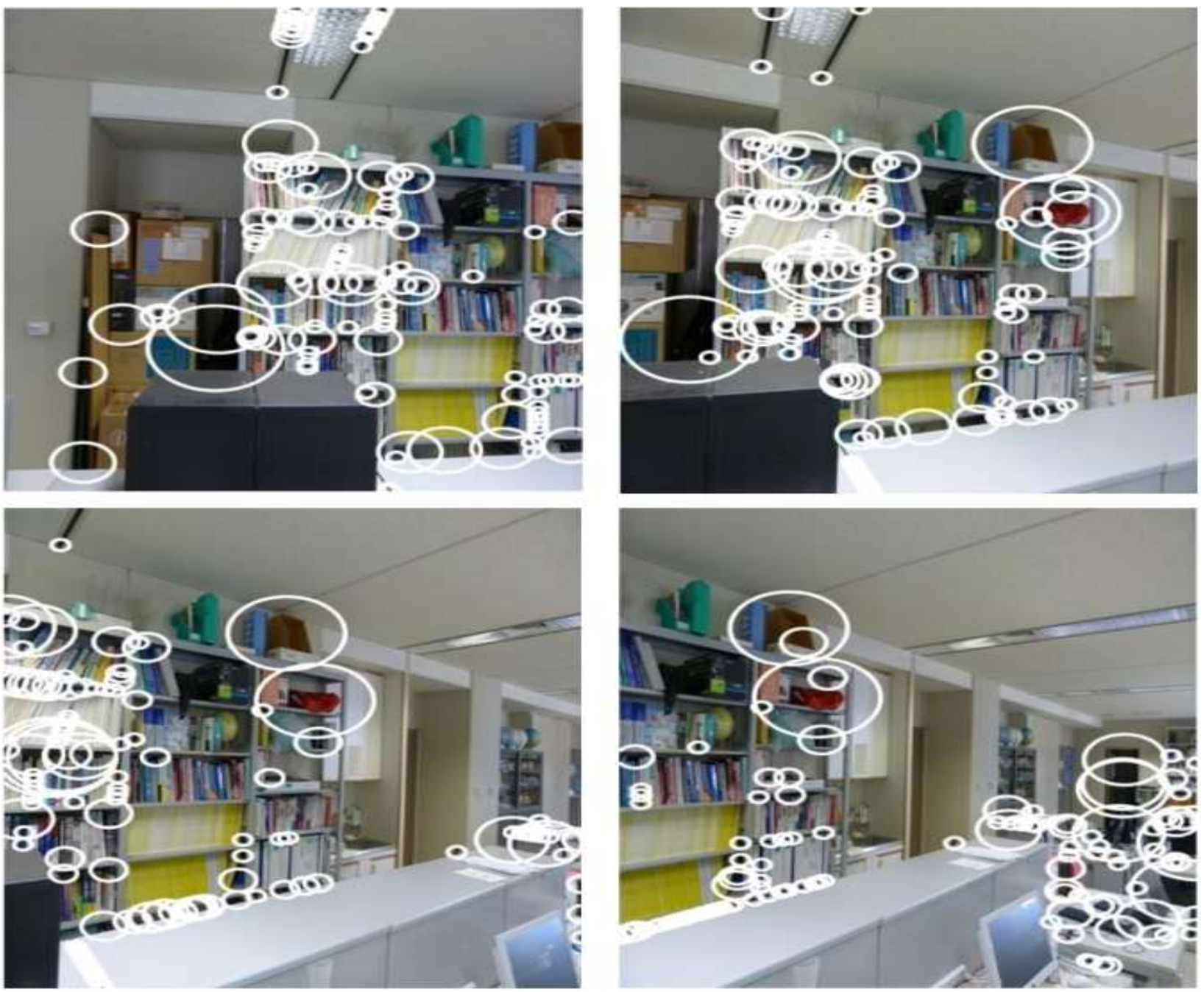

Figure 3: Example of key-point detection of input images

\section{Feature Matching by Hamming Distance}

For binary string based descriptors like BRIEFwhich is used Hamming distance as measurement. This measures the minimum number of changes to a string to another or defines the minimum number of errors that can transform a string into another.

$d^{H A D}(i, j)=\sum_{i=0}^{n-1}\left[y_{a, i} \neq y_{b, i}\right]$

In equation (3), $d^{H A D}$ is the Hamming distance between the objects $a$ and $b$, and $i$ is the index of variable of $b$ in the total number of variables $n$. The measurement process of minimum distance between two vertices using Hamming distance is shown in Figure 4.

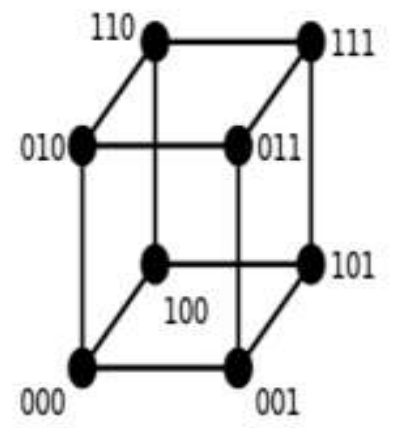

Hamming distance measured by binary 3-bit

Figure 4: Hamming distancebetween two binary strings in any vertices. 


\subsection{Characteristics of Brute-Force Matcher}

The brute-force matcher is easy to implement. According to the distance calculation, it takes the feature vector and then matched with other features. Finally, returned back to the closest one. However, it is used for SIFT, SURF etc. to better performance.

\subsection{Characteristics of FLANN based Matcher}

Fast Library for Approximate Nearest Neighbors (FLANN) can be used for searching the fast nearest neighbor in large datasets and also represented for a method of high dimensional features extractions. However, it performs the better performance comparing with Brute-Force matcher for large datasets. For binary string based descriptors like BRIEF which used Hamming distance as measurement. We proposed FLANN based matcher to get the more accurate results for using large datasets. Figure 5 shows the differences using the Brute force and FLANN based matcher method in an image.

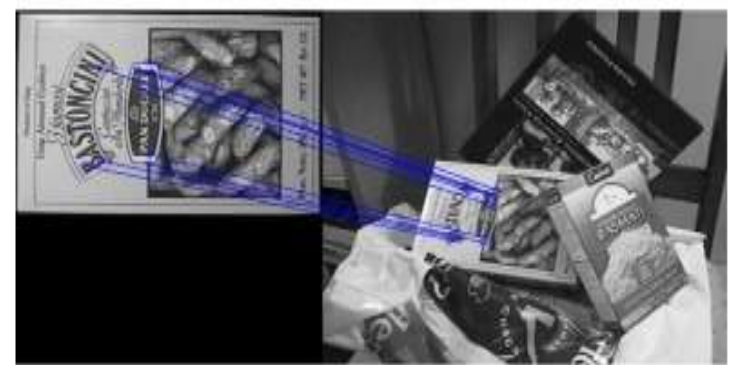

(a)

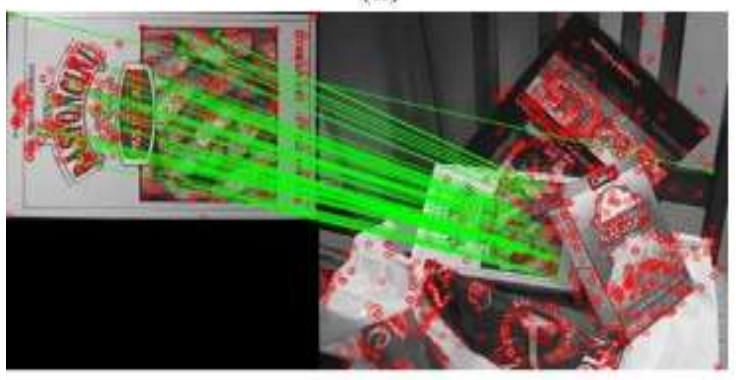

(b)

Figure 5: (a) Brute force matcher (b) FLANN based matcher

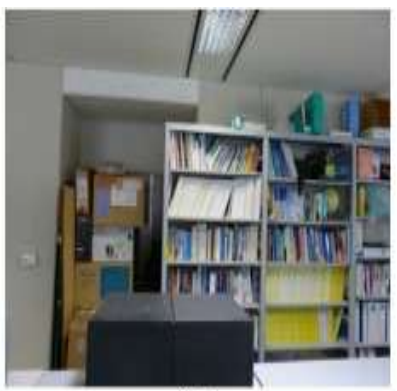

(a)

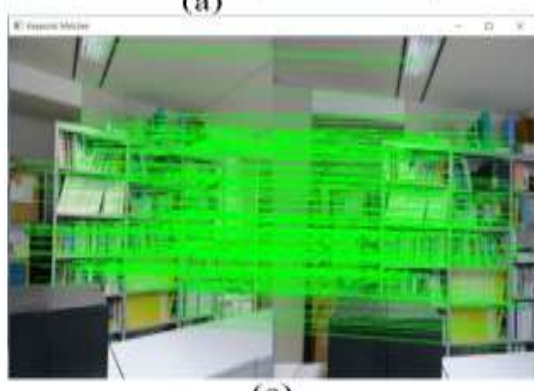

(e)

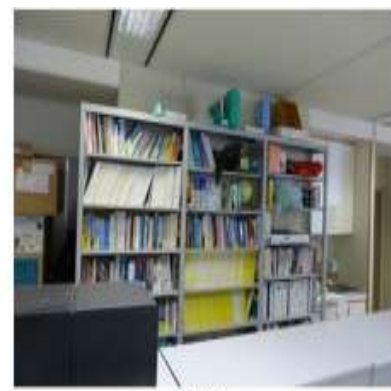

(b)

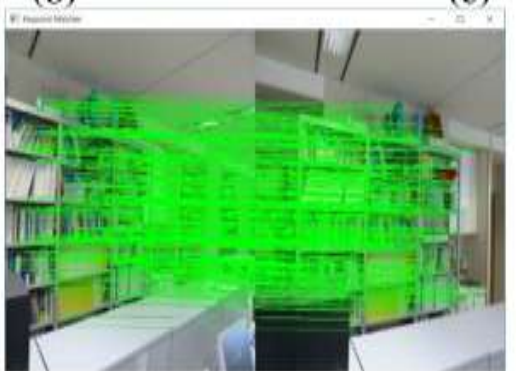

(f)

\section{Homography Estimation using RANSAC}

\subsection{Homography}

The notion of the Homography is different from the viewpoint, interpretation, and research, and especially from the perspective of differentoccupations, from the appearance of two flat objects. The Homography is the process of any two images with the same planar surface in space. However, it is familiar to the Cartesian plane, which is formed by a certain number of points which are related to the pair of real number, that is, $\mathrm{X}-\mathrm{Y}$ over two axes.In this situation, the $2 \mathrm{D}$ point can be planned in the space and designed to achieve particular aim.However, this is defined as "Homography between 2 planes", which is given only a flat 4 point, there always exists a relationship which is transformed into another equivalent of 4 points.AHomography is a $3 \times 3$ matrix we can write it as:

$H=\left[\begin{array}{lll}h_{11} & h_{12} & h_{13} \\ h_{21} & h_{22} & h_{23} \\ h_{31} & h_{32} & h_{33}\end{array}\right]$

To consider the first set of corresponding points $\left(a^{\prime}, b^{\prime}\right)$ and $(\mathrm{a}, \mathrm{b})$ for the first image and second image respectively. Then, the Homography $H$ maps them in the following way,

$\left[\begin{array}{l}a^{\prime} \\ b^{\prime} \\ 1\end{array}\right]=H\left[\begin{array}{l}a \\ b \\ 1\end{array}\right]=\left[\begin{array}{lll}h_{11} & h_{12} & h_{13} \\ h_{21} & h_{22} & h_{23} \\ h_{31} & h_{32} & h_{33}\end{array}\right]\left[\begin{array}{l}a \\ b \\ 1\end{array}\right]$

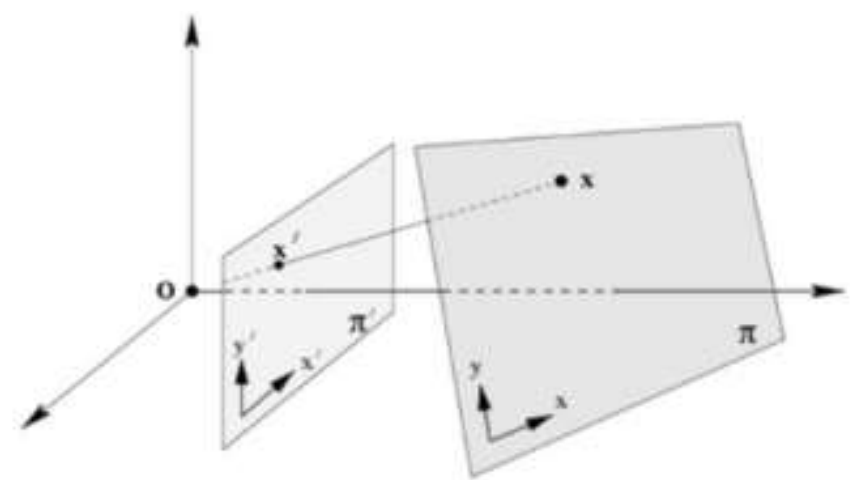

Figure 6: An example of a planar surface and the image plane.

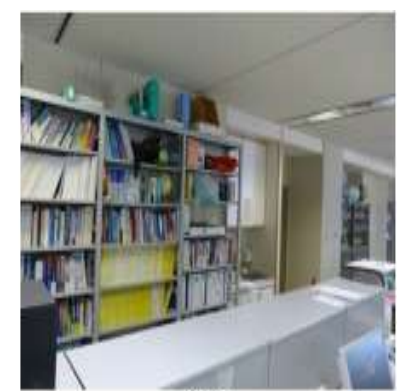

(c)

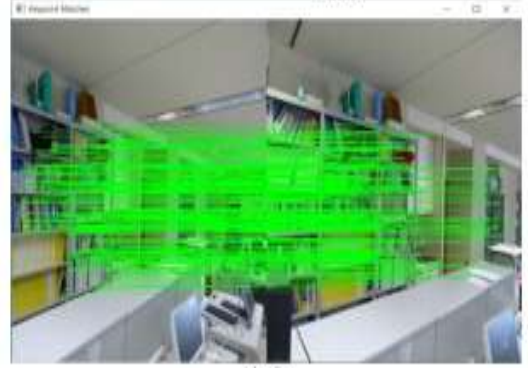

(g)

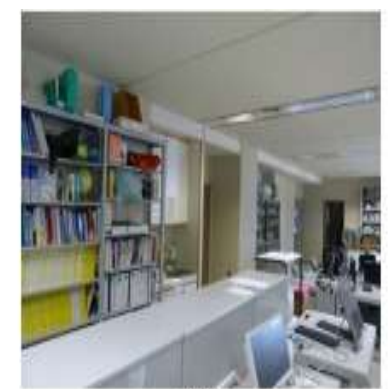

(d)

Figure7: Input images are (a), (b), (c) and (d).The Homography image between (a) and (b), (b) and (c), and (c) and (d) are (e),(f), (g) respectively. 


\section{Experiment and Results}

In the experiment, we used the images from "PASSTA Datasets [18]" and also created our own datasets. Multiples images were used to create a panorama image. Figure 8shows an examples of our system. We used multiple images as input in sequential orders, and created a panorama image.In this experiment, the input imagedimension is 384 x 512 pixels, and also we used 1488 x
1116 pixels as input imagein another example that is shown in Figure 9 .

We performed three steps for panorama stitching:

1. To ensure the quality measurement between input images for brightness fusion like color, saturation and contrast

2. Describes the feature vector from input images using BRIEF method and matched using Flann based matcher

3. Homography estimation using RANSAC algorithm [19] and create a panorama images using the Homography matrix.
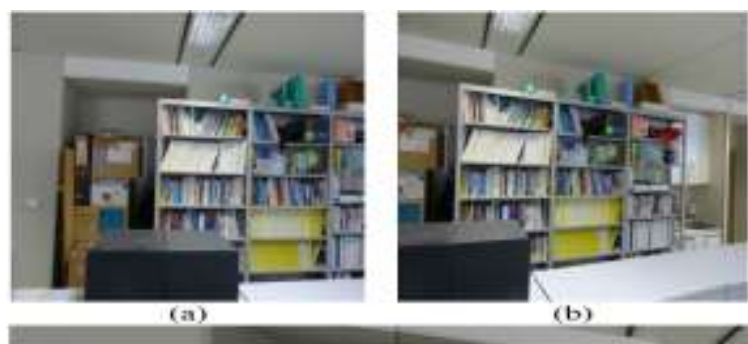

(b)

(b)

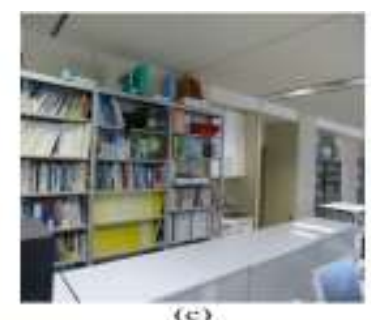

(c)

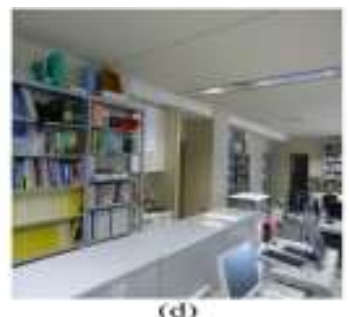

(d)

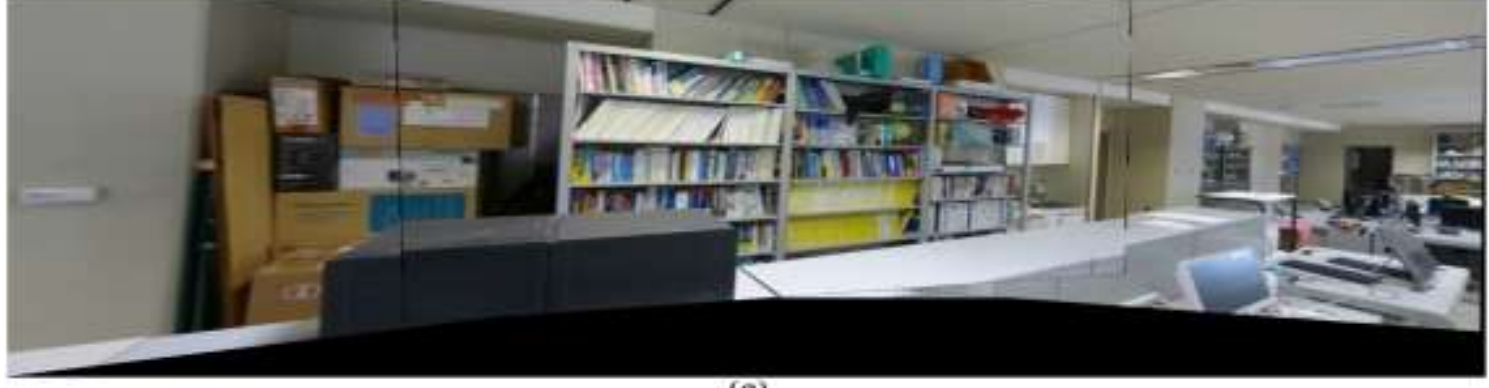

(e)

Figure8: Input image sequences are(a), (b), (c) and (d), andThe output panorama image is (e).

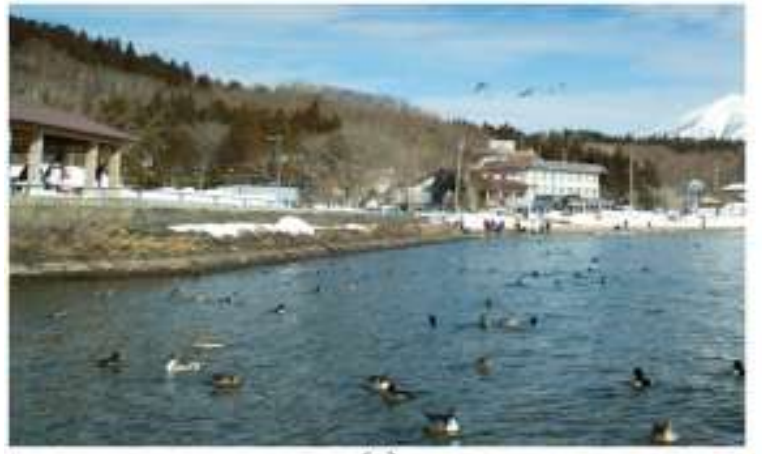

(a)

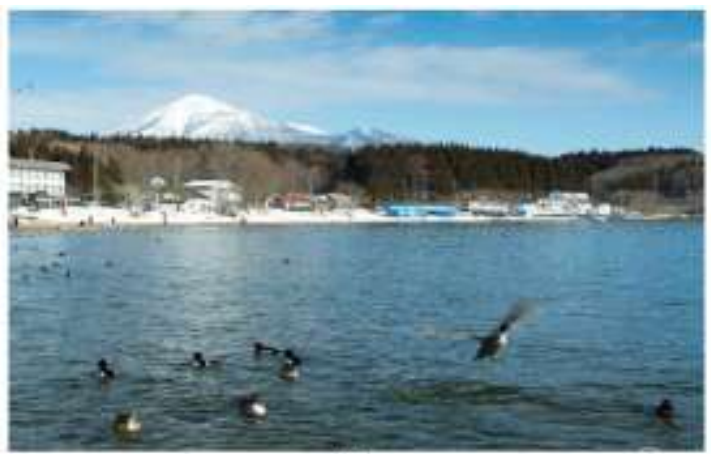

(b)

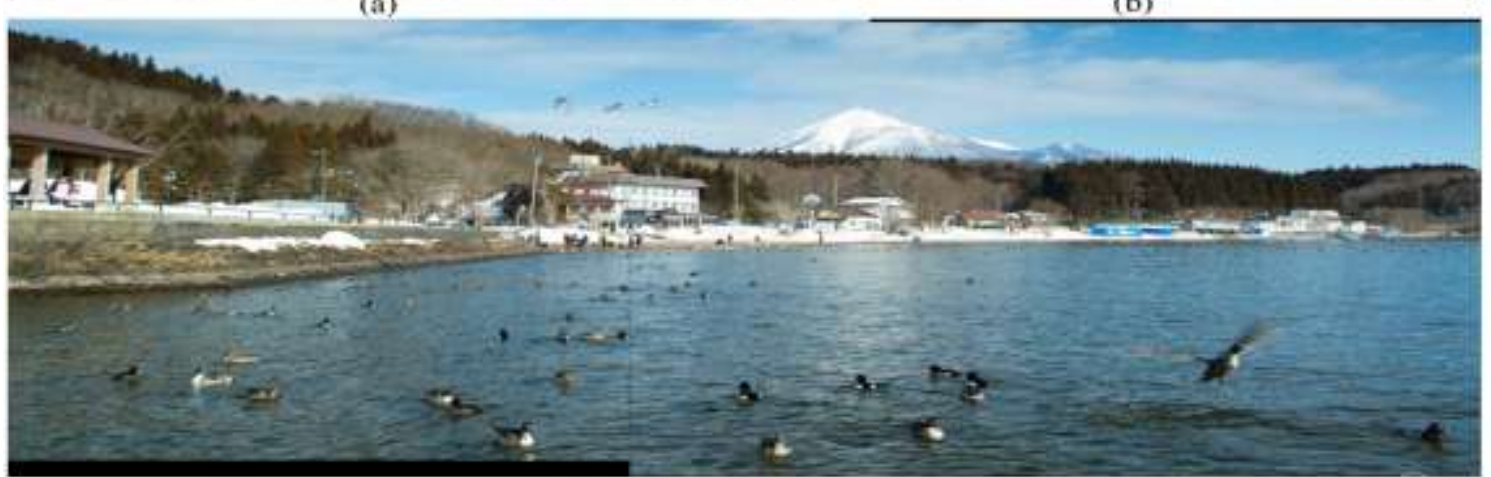

(c)

Figure9: Input images are (a) and (b), and the panorama image is (c).

\section{Conclusion}

This paper proposes a simple while efficient way for panoramic image stitching system using RANSAC with efficient brightness fusion.However, it is difficult to capture panoramic images with specific resolution using a camera. Therefore, weproposea method to improve the accuracy of the system by using binary string based descriptors, and FLANN based matchers to get more accurate results.Thus, we have collected multiples images of the whole scene, and improve the brightness fusion and combined all bits into a larger image, which is covered by a sequence of scenes. As a result, we were able to create and improve the accuracy of panorama image with efficient brightness fusion. Future work will improve the pose as an unordered collection of scene with high resolution. 


\section{References}

[1] Pranoti Kale and K.R. Singh (2015). 'A Technical Analysis of Image Stitching Algorithm', ISCIT, vol. 6, no. 1. Retrieved fromhttps://pdfs.semanticscholar.org/3554/0a11cb0d04ae53ea9c79 3b675a5b68eac768.pdf

[2] Chengming Zou, Pei Wu and Zeqian Xu (2017). "Research on Seamless Image Stitching based on Depth Map." In ICPRAM, pp. 341-350. $\quad$ Retrieved from http://www.scitepress.org/Papers/2017/61463/61463.pdf

[3] Shimon Daniel Cohen, Noga Zieber, Rotem Littman, and Udy Danino (2017). "System and method for panoramic image processing." U.S. Patent Application, 15/115, 381 Retrieved from https://patents.google.com/patent/US20170011488A1/en

[4] Ebtsam Adetet. al. (2014), "Image Stitching based on Feature Extraction Techniques: A Survey", International Journal of Computer Applications, vol 99, no 6, 2014. Retrieved from https://pdfs.semanticscholar.org/c859/ad210de8965b12bb1c9b5e2b 34ad2aa4e964.pdf

[5] Parul. M. Jain, Prof. Vijaya. K. Shandliya (2003), "A Review Paper on Various Approaches for Image Mosaicing", International Journal of Computational Engineering Research, vol 3, no. 4, pp. 106-109 Retrieved from https://pdfs.semanticscholar.org/a0e3/7be2d69719e38ead32d813d3 b8d5362118ff.pdf

[6] Matthew Brown and David G. Lowe (2007). "Automatic panoramic image stitching using invariant features." International journal of computer visionvol. 74, no. 1, pp. 59-73. Retrieved from https://link.springer.com/article/10.1007/s11263-006-0002-3

[7] Zhicheng Wang, Yufei Chen, Zewei Zhu, and Weidong Zhao (2016). "An automatic panoramic image mosaic method based on graph model." Multimedia Tools and Applications, vol. 75, no. 5, pp. 2725-2740. Retrieved fromhttps://link.springer.com/article/10.1007/s11042-015-2619-0

[8] S. Pravenaa and R. Menaka (2016). "A methodical review on image stitching and video stitching techniques." International Journal of Applied Engineering Research, vol. 11, no. 5, pp. 3442-3448 Retrieved fromhttp://www.ripublication.com/ijaer16/ijaerv11n5_80.pdf

[9] Shreyas Mistry and Arpita Patel (2016). "Image Stitching using Harris Feature Detection." International Research Journal of Engineering and Technology (IRJET), vol. 3, no. 04, pp. 2220 2226 Retrieved from https://irjet.net/archives/V3/i4/IRJETV3I4270.pdf

[10] David G. Lowe (2004). "Distinctive Image Features from Scale Invariant Key-points", International Journal of Computer Vision, vol. $60 \quad$ Retrieved from https://www.robots.ox.ac.uk/ vgg/research/affine/det_eval_files/lo we_ijcv2004.pdf

[11] Weijie Huang, and Xiaowei Han (2013). "An improved RANSAC algorithm of color image stitching." In Proceedings of 2013 Chinese intelligent automation Conference, pp. 21-28. Springer, Berlin, Heidelberg, 2013. Retrieved fromhttps://link.springer.com/chapter/10.1007/978-3-642-38466$0 \_3$

[12] Jing Zhang, Guangxue Chen and Zhaoyang Jia (2017). "An image stitching algorithm based on histogram matching and SIFT algorithm." International Journal of Pattern Recognition and Artificial Intelligence, vol. 31, no. 04, pp. 1754006. Retrieved from

http://www.worldscientific.com/doi/abs/10.1142/S0218001417540 064

[13] Diptiben Patel, Bhoomika Sonane, and Shanmuganathan Raman (2017). "Multi-exposure Image Fusion Using Propagated Image Filtering." In Proceedings of International Conference on Computer Vision and Image Processing, pp. 431-441. Retrieved from https://link.springer.com/chapter/10.1007/978-981-10-2104-

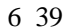

[14] Yu Tang and Huiyan Jiang (2009). "Highly efficient image stitching based on energy map." In Image and Signal Processing, CISP'09. 2nd International Congress on, $\mathrm{pp}$. 1-5. Retrieved fromhttp://ieeexplore.ieee.org/abstract/document/5304214/

[15] Yu Tang and Jungpil Shin (2010). "De-ghosting for image stitching with automatic content-awareness." In Pattern Recognition (ICPR), 20th International Conference on, pp. 2210-2213. Retrieved from http://ieeexplore.ieee.org/abstract/document/5595971/

[16] Yu Tang and Jungpil Shin (2014). "Image stitching with efficient brightness fusion and automatic content awareness." In Signal Processing and Multimedia Applications (SIGMAP), International Conference on IEEE, pp. 60-66. Retrieved fromhttp://ieeexplore.ieee.org/abstract/document/7514477/

[17] Yuanzhen Li, Lavanya Sharan and Edward H. Adelson (2005). "Compressing and Companding High Dynamic Range Images with Subband Architectures", ACM Transactions on Graphics, vol. 24 no. 3, pp. 836-844 Retrieved from http://www.mit.edu/ yzli/hdr05.pdf

[18] "PASSTA Datasets", Retrieved fromhttp://www.cvl.isy.liu.se/en/research/datasets/passta/.

[19] M. A. Fischler, R. C. Bolles (1981). "Random Sample Consensus: A Paradigm for Model Fitting with Applications to Image Analysis and Automated Cartography", Comm. of the ACM, vol 24, pp 381395.Retrieved

fromhttps://www.sri.com/sites/default/files/publications/ransacpublication.pdf 